\title{
Preparation, Characterization and Catalytic Activity of Palladium Nanoparticles Embedded in the Mesoporous Silica Matrices
}

\author{
Nadiia A. Ivashchenko ${ }^{*}$, Wojciech Gac $^{2}$, Valentyn A. Tertykh ${ }^{1}$, Viktor V. Yanishpolskii ${ }^{1}$, \\ Sergei A. Khainakov ${ }^{3}$, Alla V. Dikhtiarenko ${ }^{3}$, Sylwia Pasieczna-Patkowska ${ }^{2}$, Witold Zawadzki ${ }^{2}$ \\ ${ }^{1}$ Chuiko Institute of Surface Chemistry of National Academy of Sciences of Ukraine, Kyiv, Ukraine \\ ${ }^{2}$ Faculty of Chemistry, Maria Curie-Skłodowska University, Lublin, Poland \\ ${ }^{3}$ Department of Organic and Inorganic Chemistry, Faculty of Chemistry, University of Oviedo, Oviedo, Spain \\ Email: ${ }^{*}$ nadiia.iva@gmail.com
}

Received April 9, 2012; revised May 4, 2012; accepted August 21, 2012

\begin{abstract}
Novel in-situ reduction approach was applied for the synthesis of palladium nanoparticles in the pores of mesoporous silica materials with grafted silicon hydride groups. Matrices possessing different structural properties (MCM-41, SBA-15 and Silochrom) were used. Samples were studied by nitrogen adsorption-desorption method, low-angle X-ray diffraction, transmission electron microscopy (TEM) and FT-IR/PAS spectroscopy. The temperature-programmed oxidation (TPO) and reduction (TPR) methods were applied to examine reducibility of palladium species. Palladium containing catalysts were tested in methane oxidation reaction. It was demonstrated that relatively large pores in SBA-15 type silica facilitated formation of well-dispersed palladium nanoparticles confined in the pores channels. In the case of MCM-41 support, metallic palladium nanoparticles were formed on the external surface. The obtained materials showed high catalytic activity. Lower activity of the samples containing small crystallites located in the pore volume at high temperatures was related to worse accessibility of active sites to the reation mixture.
\end{abstract}

Keywords: Palladium Nanoparticles; Siliconhydride Groups; Mesoporous Ordered Silica; MCM-41; SBA-15; Palladium-Containing Nanocomposites; Methane Oxidation

\section{Introduction}

Metal nanoparticles have attracted considerable attention in modern technology. The emerging physicochemical properties of materials are often observed when the particle size reaches nanometer range [1]. Palladium nanoparticles are expected to show high activity and selectiveity in numerous catalytic processes.

The application of unsupported metal nanoparticles is often limited due to aggregation processes which occur in the activation stage or catalytic reaction conditions. Nowadays significant number of research studies has been devoted to the synthesis of such objects with searching for possible ways to prevent undesirable sintering processes [2-4]. The use of advantageous porous supports, which can decrease the growth of particles is one of the approaches for the control of metal nanoclusters during synthesis and remaining them separated from each other, even at high temperatures [2,4-8]. However, application of common reducing reagents, such as sodium borohydride, hydrazine [5,9] or hydrogen

*Corresponding author.
[6] usually leads to considerable clusters aggregation in the synthesis conditions.

Immobilization of groups possessing pronounced reducing properties on the surface of silica allow one to solve the problem of nanoparticles sintering. In this case, formation of nanoparticles on the silica surface occurs due to reduction of metal ions immediately in a place of reducer attachment [10]. Reduction process is caused by properties of surface silicon hydride groups, accompanied by their hydrolysis and formation of high-disperse metal particles of nanometer size $[11,12]$.

Recently silica materials with grafted silicon hydride groups were successfully applied for the synthesis of gold and silver nanoparticles [13-15]. Direct reduction of ions on the surface of hydridesilica, in contrast to common reduction in the hydrogen atmosphere, allowed one to regulate the size of the formed metal particles within certain limits, by varying concentration of metal salt taken for reduction and time of reduction.

Thereby in present work we combine two advantageous approaches. Silicon hydride groups, possessing reducing properties, grafted to the surface of silica allow 
obtaining metal nanoparticles immediately in the place of reducer attachment. Another consists in application of ordered mesoporous silicas as supports confining palladium nanoparticles and preventing particles aggregation. The palladium/silica nanocomposites were characterized by different physico-chemical methods and tested as catalysts in methane oxidation reaction.

Methane is a relatively potent greenhouse gas. About $8 \%$ of the world's anthropogenic methane emissions come from coal mines around the world. The catalytic oxidation of methane may be considered as a promising solution of methane-poor gas mixtures utilization. The process allows not only to achieve methane complete oxidation in its low-concentrated mixtures, but it also may be carried out at moderate (depended on the catalyst used) temperatures [16]. Noble metals, especially palladium, placed on the supports with large specific surface areas and good thermal stability, demonstrated the highest activity for complete oxidation of methane among other $[17,18]$.

\section{Experimental}

\subsection{Samples Preparation}

Silicon hydride groups immobilized on the surface of mesoporous ordered silica (MCM-41, SBA-15) and silochrom type silica were used for in situ reduction of palladium ions. Silochrom is a porous material obtained from concentrated water suspensions of fine pyrogenic silica (Aerosil).

For the preparation of mesoporous ordered silica materials (MCM-41 and SBA-15) following procedures were applied. In the synthesis of MCM-41 material reaction mixture with the mole ratio 1.0TEOS:0.153CTM$\mathrm{AB}: 1.57 \mathrm{NH}_{4} \mathrm{OH}: 148 \mathrm{H}_{2} \mathrm{O}$ was used according to the recommendations given by Grun et al. [19]. In the synthesis $8.3 \mathrm{~g}$ of hexadecyltrimethylammonium bromide (CTAB) was dissolved in $270 \mathrm{~cm}^{3}$ of distilled water. The mixture was stirred at the temperature $40^{\circ} \mathrm{C}$, and then 24 $\mathrm{cm}^{3}$ of tetraethylortosilicate (TEOS) was introduced. Next $24 \mathrm{~cm}^{3}$ of ammonium hydroxide (25\%) was slowly introduced. The obtained white gel was stirred for $1 \mathrm{~h}$, and then filtered and washed with distilled water. A sample was dried for 9 hours at $90^{\circ} \mathrm{C}$, and then initially calcined at the static air at $550^{\circ} \mathrm{C}$ for $6 \mathrm{~h}$.

SBA-15 mesoporous silica powder was prepared according to the procedure reported in the study made by Zhao et al. [20]. $20 \mathrm{~g}$ of poly(ethylene oxide-propylene oxide-ethylene oxide) triblock copolymer with the general formula $\mathrm{EO}_{20} \mathrm{PO}_{70} \mathrm{EO}_{20}$ (Pluronic P123) was dissolved in $600 \mathrm{~cm}^{3}$ of distilled water and $162 \mathrm{~cm}^{3}$ of $2 \mathrm{M}$ $\mathrm{HCl}$. The mixture was stirred for 5 hours at the temperature $38^{\circ} \mathrm{C}$. Next $39.4 \mathrm{ml}$ of TEOS was poured to the flask and the solution was stirred for 20 more hours at the same temperature. The resulting mixture was then heated in thermostat at $100^{\circ} \mathrm{C}$ for $24 \mathrm{~h}$. The white product was filtrated, washed, dried, and calcinated at $550^{\circ} \mathrm{C}$ for $6 \mathrm{~h}$.

Modification of silica matrix was carried out under anhydrous conditions according to the procedure previously described in [21]. Triethoxysilane was added dropwise to glacial acetic acid. After stirring, mixture was poured to the flask containing silica. After impregnation, the obtained mixture was transferred to the thermostat and heated there for $2 \mathrm{~h}$ at $90^{\circ} \mathrm{C}$. Samples of modified silica were dried in an air at $90^{\circ} \mathrm{C}$ during one hour and then heated in the oven at $150^{\circ} \mathrm{C}$ for $2 \mathrm{~h}$.

Introduction of palladium was performed by impregnating of modified silica with palladium nitrate solution with $\mathrm{pH}=1.6$. Samples were then washed with distilled water, and dried at $100^{\circ} \mathrm{C}$ for 3 hours. Metal nanoclusters were formed due to reduction of palladium ions immediately in a place of surface $\equiv \mathrm{SiH}$ groups attachment.

Palladium loading in as-prepared composites was the same $0.5 \%$, however samples were prepared using different silica supports, so they were denoted as $\mathrm{Pd} / \mathrm{Si}$ lochrom, Pd/MCM-41, and Pd/SBA-15. Control of the metal content in the equilibrium solution was carried out by spectrophotometric method with the use of thiourea [22]. Measurements were carried out by spectrophotometer SF 46 (LOMO, Russia) at the wavelength 460 $\mathrm{nm}$ using cuvettes with layer thickness $1 \mathrm{~cm}$. Content of palladium in one gram of $\mathrm{Pd} /$ Silica nanocomposite (wt\%) was determined as it was reported in [21,23].

In order to remove the residual acetic acid used for modification and nitrate species, all the samples before analyses were heated up to $370^{\circ} \mathrm{C}$ in the helium flow (50 $\left.\mathrm{cm}^{3} / \mathrm{min}\right)$.

\subsection{Samples Characterization}

The analysis of samples low-temperature adsorption/ desorption of nitrogen were obtained using analyser of porosity and specific surface area Micromeritics ASAP 2020 , USA at $-196^{\circ} \mathrm{C}$. Total surface area was calculated from BET method and pore volume as well as pore size distribution was obtained using BJH method.

$\mathrm{X}$-ray powder diffraction analysis was carried out with DRON-4-07 X-ray diffractometer (Burevestnik, Russia) using a Ni-filtered $\mathrm{Cu} K_{\alpha}$ radiation.

TEM observations were performed on MET JEOL2000 EX-II (Japan) transmission electron microscope. For the TEM analysis, the materials were dispersed in ethanol and a drop of this suspension was deposited onto a carbon-coated copper grid.

FT-IR/PAS spectra of the samples were recorded by means of the Bio-Rad Excalibur 3000 MX spectrometer equipped with photoacoustic detector MTEC300 (in the helium atmosphere in a detector) over the $4000-400$ $\mathrm{cm}^{-1}$ range at the resolution of $4 \mathrm{~cm}^{-1}$ and maximum source aperture. The spectra were normalized by comput- 
ing the ratio of a sample spectrum to the spectrum of a MTEC carbon black standard. A stainless steel cup (diameter $10 \mathrm{~mm}$ ) was filled with powder samples (thickness $<6 \mathrm{~mm}$ ). Interferograms of 1024 scans were averaged for each spectrum.

In the temperature-programmed oxidation (TPO) analysis, the sample was introduced to the quartz flow reactor, then the temperature of the reactor was increased with the rate $10^{\circ} \mathrm{C} / \mathrm{min}$. The total flow of $5 \% \mathrm{O}_{2} / \mathrm{He}$ mixture was $30 \mathrm{~cm}^{3} / \mathrm{min}$. Evolved gases were analyzed by mass spectrometer HAL201RC (Hiden Analytical).

Reducibility of palladium species was determined by temperature-programmed reduction method (TPR) using apparatus AMI-1 (Altamira Instruments Inc.). The analysis was carried out by placing $0.05 \mathrm{~g}$ of the sample in a quartz reactor. Reduction was performed in the mixture $6 \% \mathrm{H}_{2} / \mathrm{Ar}$ at the flow rate $30 \mathrm{~cm}^{3} / \mathrm{min}$, the linear temperature increase was $10^{\circ} \mathrm{C} / \mathrm{min}$. The samples before reduction were pre-treated in the flow of argon (30 $\mathrm{cm}^{3} / \mathrm{min}$ ). Samples were heated up to $370^{\circ} \mathrm{C}$, kept at this temperature for 30 minutes and then cooled down to $-70^{\circ} \mathrm{C}$. Water vapor formed during reduction was removed in a cold trap (immersed in liquid nitrogen-methanol slush). The signal of thermal conductivity detector (TCD) was calibrated by injecting $55 \mu$ l of argon to the carrier gas.

The activity of the catalysts in the complete oxidation of methane was determined in the quartz reactor filled

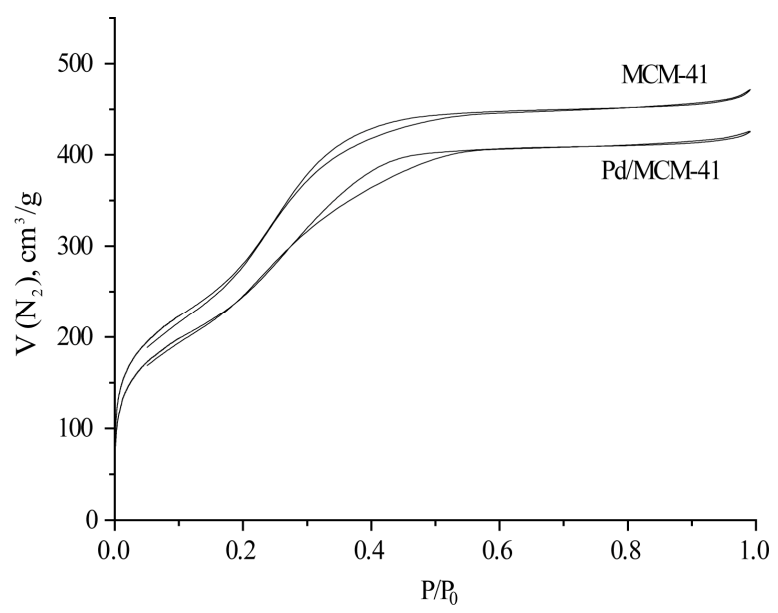

(a) with the $0.05 \mathrm{~g}$ of catalyst. The reaction mixture was composed of $1 \% \mathrm{CH}_{4}$ and $99 \%$ air. The total flow rate of the mixture was $100 \mathrm{~cm}^{3} / \mathrm{min}$. Temperature was increased stepwise at $20^{\circ} \mathrm{C}$ every $10 \mathrm{~min}$ from $150^{\circ} \mathrm{C}$ up to $700^{\circ} \mathrm{C}$.

\section{Results and Discussion}

The nitrogen adsorption/desorption isotherms for MCM41, SBA-15 and palladium-supported materials are displayed in Figure 1. The isotherms of the samples exhibited a highly ordered mesoporous nature, whereas the sharp capillary condensation step in the range $p / p_{0}=0.2$ - 0.4 for MCM-41 and $p / p_{0}=0.6-0.8$ for SBA-15 suggests cylindrical pores with narrow pore size distri- bution. The isotherms of palladium-containing nanocomposites (Pd-MCM-41 and Pd-SBA-15) indicate that metal introduction doesn't affect the ordered mesoporous structure of the supports; however, volume of adsorbed nitrogen is lower than in the case of initial silica matrix.

Specific surface area $\left(S_{\mathrm{BET}}\right)$ and pore volume $\left(V_{\text {pores }}\right)$ of palladium containing nanocomposite decreases in comparison with initial silica matrix SBA-15. The textural properties of the initial ordered silicas and palladiumcontaining composites are summarized in Table $\mathbf{1 .}$

The location palladium nanoparticles on the external surface or in the pore channels of silica matrices may

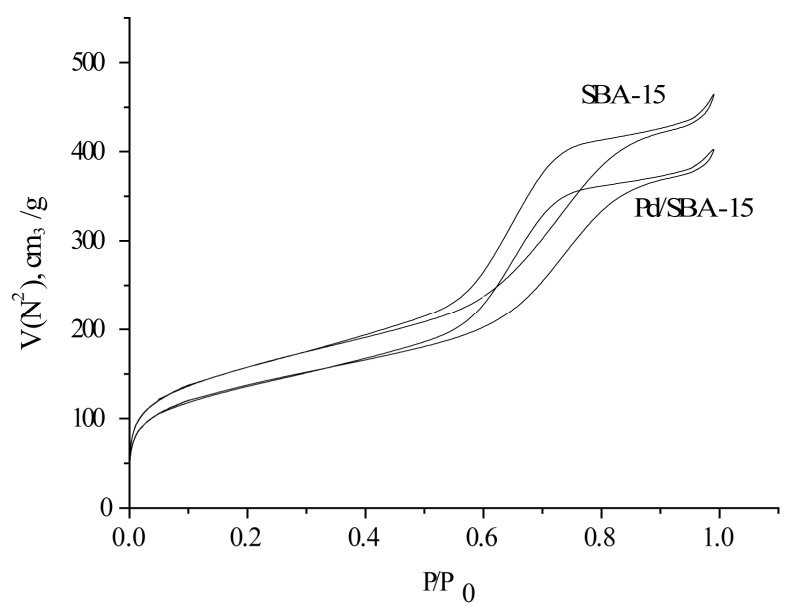

(b)

Figure 1. Isotherms of low-temperature ad(de)sorption of nitrogen at $-196^{\circ} \mathrm{C}$ for initial silicas (a: MCM-41; b: SBA-15) and palladium-containing composites (Pd/MCM-41, Pd/SBA-15).

Table 1. Structural characteristics of initial silica matrices and palladium-containing nanocomposites based on them.

\begin{tabular}{cccccc}
\hline Parameter & Silochrom & Pd/Silochrom & SBA-15 & Pd/SBA-15 & MCM-41 \\
\hline $\mathrm{d}_{\text {pores }}, \mathrm{nm}$ & 17 & 20 & 6.2 & 6.2 & 2.3 \\
$S_{B E T}, \mathrm{~m}^{2} / \mathrm{g}$ & 120 & 101 & 540 & 473 & 939 \\
$V_{\text {pores }}, \mathrm{cm}^{3} / \mathrm{g}$ & 0.975 & 0.722 & 0.574 & 0.497 & 0.530 \\
\hline
\end{tabular}


block nitrogen flow into the pores, leading to the decrease of adsorbed gas quantity causing a shortage of specific surface area and pore volume. Thus, in the case of $\mathrm{Pd} / \mathrm{SBA}-15$ pores volume is reduced by $13.4 \%$ and the shortage of specific surface area is $12.4 \%$, for the $\mathrm{Pd} / \mathrm{MCM}-41$ those values are 9 and $11 \%$ correspondingly, and $26 \%$ and $16 \%$ for the $\mathrm{Pd} /$ Silochrom sample.

Figure 2(a) shows the small-angle XRD pattern of the MCM-41 mesoporous silica powder and Pd/MCM-41 nanocomposite. The small angle XRD pattern for MCM41 shows four well-resolved peaks which can be indexed as (100), (110), (200) and (210) reflections and are characteristic for hexagonal porous structure. TEM image for MCM-41 type silica matrix (Figure 3(a)) demonstrates parallel porous channels typical for it. Four reflections displayed in the small-angle region of XRD spectrum

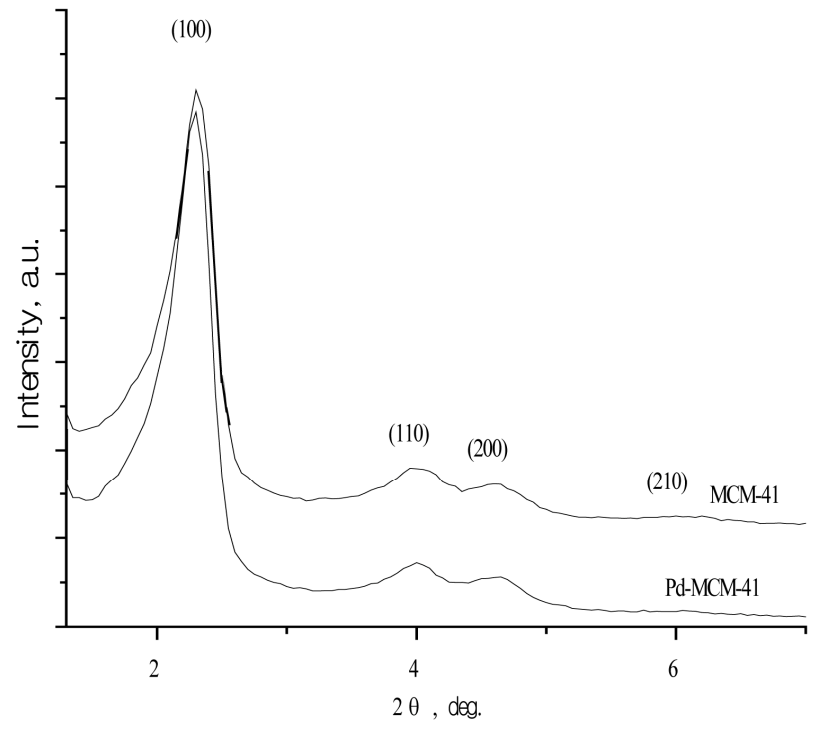

(a) for $\mathrm{Pd} / \mathrm{MCM}-41$ nanocomposite indicate that hexagonal phase of silica matrix remains intact after impregnation of MCM-41 support with grafted $\mathrm{SiH}$ groups with palladium nitrate solution Figure 2(a). This can be explained by the formation of palladium nanoparticles on the external surface of support, as it can be seen from TEM image for $\mathrm{Pd} / \mathrm{MCM}-41$ nanocomposite (Figure 3(b)). Formed particles have quite large size and broad distribution (from 10 up to $50 \mathrm{~nm}$ ).

Diffraction pattern in the small-angle region of synthesized silica SBA-15 type Figure 2(b) demonstrates tree well-resolved peaks assigned to (100), (110) and (200) reflections, which suggest hexagonal ordered structure of support. Transmission electron microscopy data confirm ordered pore array in the synthesized matrices, honeycomb porous structure characteristic for SBA-15, is easily

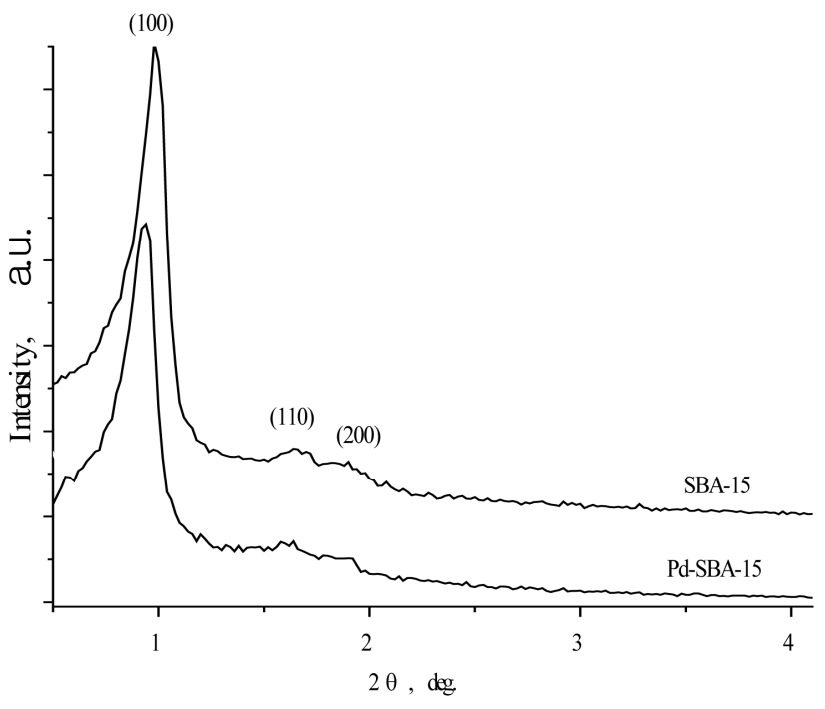

(b)

Figure 2. Small-angle X-ray diffraction pattern of initial ordered mesoporous silica matrices (a: MCM-41; b: SBA-15) and palladium-containing composites (Pd/MCM-41, Pd/SBA-15).
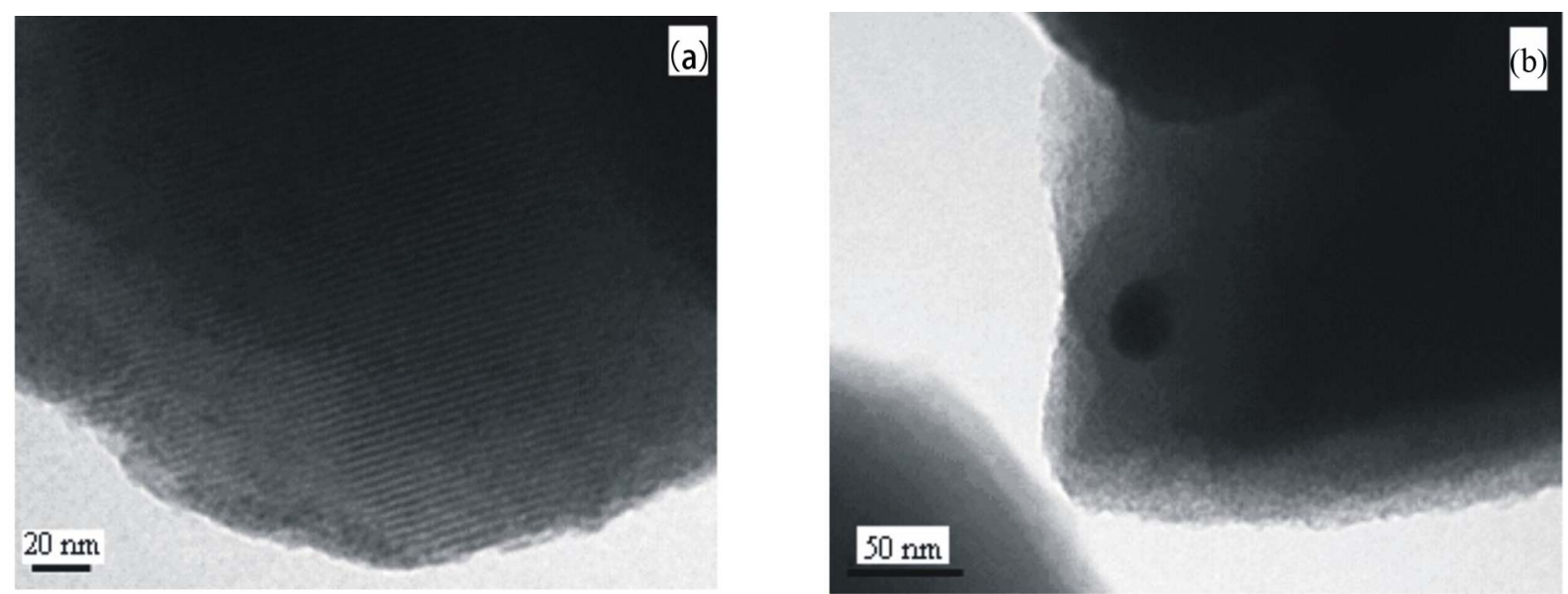

Figure 3. TEM images of silica support MCM-41 type (a) and palladium nanoparticles supported on it (b). 
seen on the image (Figure 4(a)). XRD investigations for Pd/SBA-15 composite revealed the decrease of diffracttion peak intensity comparing to supports diffraction pattern (Figure 2(b)). This change can be related to a partial distortion of the regular structure caused by metal incorporation in the pore channels of SBA-15, however, small content of palladium in composite $(0.5 \%)$ does not lead to complete destruction of structure and intensity decrease is quite small.

Transmission electron microscopy data confirm the formation of palladium nanoparticles inside the pore channels of SBA-15, as well as retention of porous structure (Figures 4(b) and (c)). Metal particles in the $\mathrm{Pd} / \mathrm{SBA}-15$ composite have narrow size distribution with the maximum near $5 \mathrm{~nm}$. Growth of nanoparticles can be limited by pores of ordered mesoporous silicas, leading to the formation of small particles with narrow size distribution.

The effects caused by palladium introduction can be also observed in the FT-IR results presented in the Figure 5. A sharp peak at $3743 \mathrm{~cm}^{-1}$ is connected with vibration of the isolated Si-OH silanol groups. Slight shoulder with the center located at around $3650 \mathrm{~cm}^{-1}$ is often ascribed to the bridging hydroxyls (SiOH-OSi) vibrations. Stretching vibrations $\nu \mathrm{OH}(\mathrm{Si}-\mathrm{O}-\mathrm{H})$ are visible in the broad peak with the center at around $3440 \mathrm{~cm}^{-1}$. Small peaks located at the shoulder of the broad band are assigned to the asymmetric $\mathrm{CH}_{3}, \mathrm{CH}_{2}$ and symmetric $\mathrm{CH}_{2}+\mathrm{CH}_{3}$ vibrations. The absorption bands at around 1030 and $1080 \mathrm{~cm}^{-1}$ are due to asymmetric stretching vibrations of Si-O-Si bridges. The absorption band at 960 - $970 \mathrm{~cm}^{-1}$ is connected with the stretching vibrations of the $\mathrm{Si}-\mathrm{OH}$ groups, while the bands at $780-800 \mathrm{~cm}^{-1}$, and $540-560 \mathrm{~cm}^{-1}$ are the symmetric stretching vibrations of Si-O-Si bridges. The bands located at $450-460 \mathrm{~cm}^{-1}$ are assigned to the $\mathrm{Si}-\mathrm{O}$ bending vibrations.

Large intensity of the peaks in the $2800-4000 \mathrm{~cm}^{-1}$ region for SBA-15 (Figure 5(b)) sample reveals a substantial amount of hydroxyl groups located in the pores. While the broadening of the peaks located between 1000 and $1300 \mathrm{~cm}^{-1}$ in SBA-15 support, indicates less ordered structure of materials.

An introduction of palladium does not strongly influence the number of hydroxyl groups nor the silica structure for $\mathrm{Pd} / \mathrm{MCM}-41$ composite (Figure 5(a)), however in the case of Pd/SBA-15 sample content of hydroxyl groups is obviously decreased comparing to initial SBA15 silica (Figure 5(b)).

In the previous research [21], it was demonstrated that silicon hydride groups grafted on the silica support may induce in-situ reduction of palladium species to metallic particles. Thus, adsorption bands at $2240 \mathrm{~cm}^{-1}$ in the FTIR spectrum of the modified silica provide evidence for the fact of silicon hydride groups' attachment. The
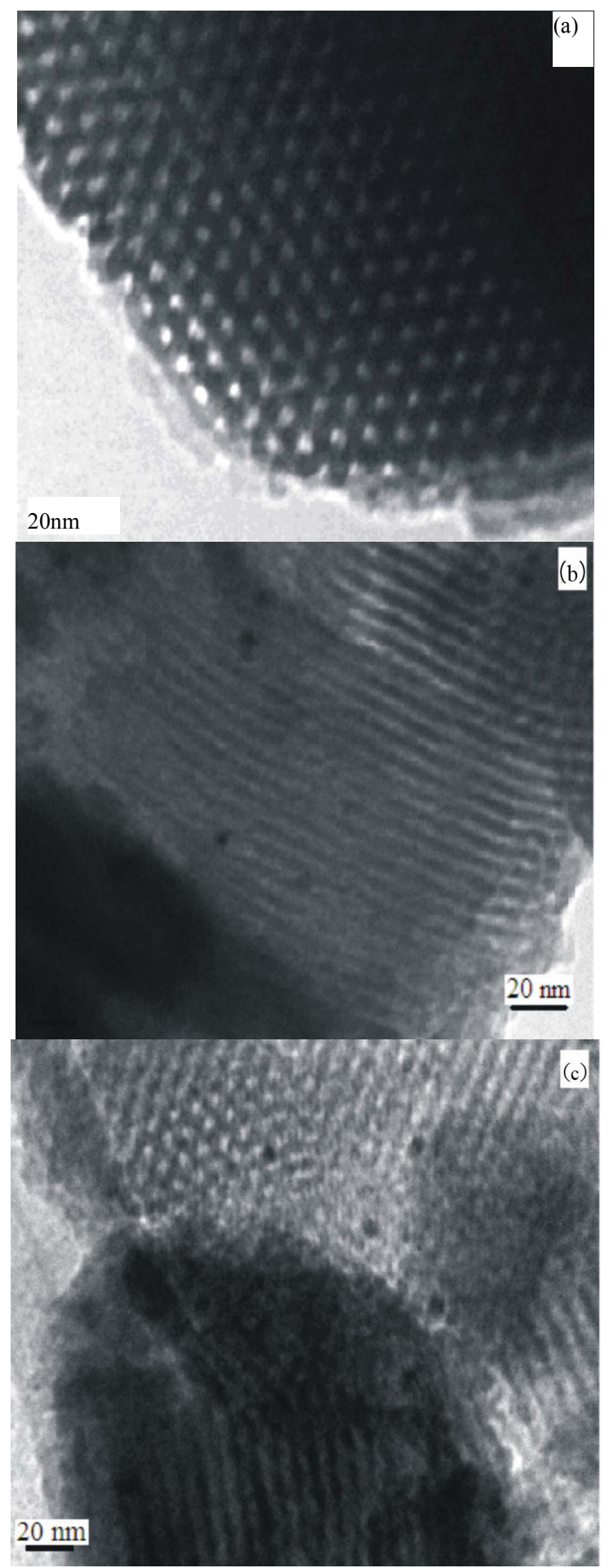

Figure 4. TEM images of silica support SBA-15 type (a) and palladium-containing nanocomposite Pd/SBA-15 (b, c).

presence of such groups in the confined space in pores of that materials as SBA-15 can lead to the formation of small palladium nanoparticles.

For the comparison, our previous investigation showed [21] that in the case of Pd/Silochrom composite suppor- 


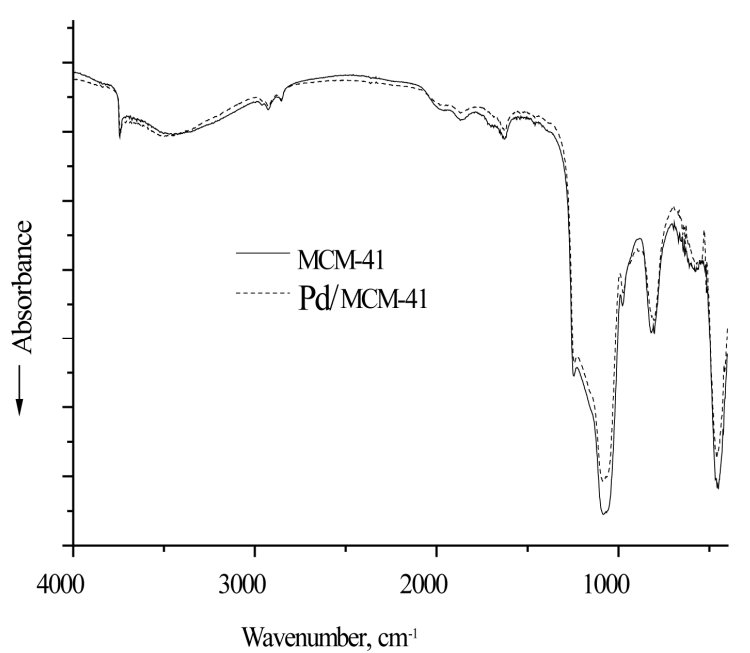

(a)

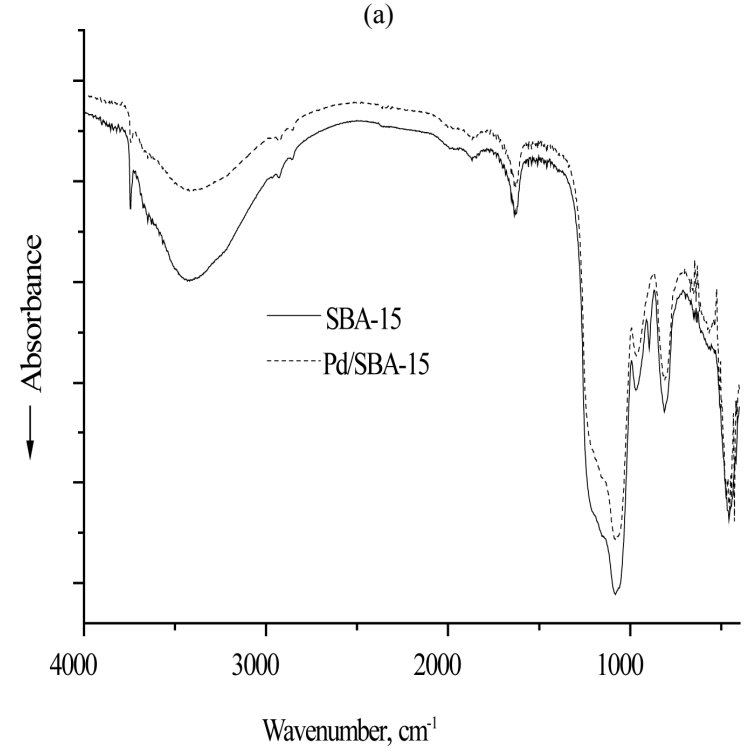

(b)

Figure 5. FT-IR spectra of the silica matrices and palladium containing composite based on it: a: MCM-41 and Pd/MCM41; b: SBA-15 and Pd/SBA-15 samples.

ted nanoparticles have size about $10-20 \mathrm{~nm}$.

Structural characteristics of supports can serve as an explanation of differences in nanoparticles arrangment in the porous structure of MCM-41 and SBA-15. Small pore diameter on MCM-41 type silica (2.3 nm in comparison to 6.2 for SBA-15) can be the reason why in $\mathrm{Pd} / \mathrm{MCM}-41$ nanocomposite palladium nanoparticles are formed mainly on the external surface of silica and none of them are incorporated in porous channels of silica matrix. Pore openings seem to be small for penetration of modificator (triethoxysilane), which at low concentrations in acetic acid media exists predominantly in the form of oligomers, into the pore channels, which leads to impossibility of particles formation directly within the pore channels.
Study of fresh composite Pd/MCM-41 by temperatureprogrammed oxidation (TPO) method reveal desorption and decomposition of compounds confined in the porous material (Figure 6). The curves indicate that the sample contains a large amount of water. Water is desorbed in the wide range of temperatures, ranged from room temperature to around $250^{\circ} \mathrm{C}$. A few overlapped peaks can be distinguished. The maxima on the $\mathrm{m} / \mathrm{e}=44$ curve located at $210^{\circ} \mathrm{C}$ and $290^{\circ} \mathrm{C}$ evidence the presence of oxidation of carbon containing compounds towards $\mathrm{CO}_{2}$. Small maxima on the curves with the pattern $\mathrm{m} / \mathrm{e}=30$ may indicate on desorption of the traces of residual nitrogen-containing compounds from palladium precursor.

The treatment conditions may be crucial for development of active phase in catalysts. Figure 7 show TPR results of the samples heated up in the flow of oxygen and argon. TPR curves of the nanocomposite Pd/MCM41 after treatment in oxygen reveal a reduction of palladium oxide species. The maximum of hydrogen consumption peak is observed at temperature ca. $60^{\circ} \mathrm{C}$ (Figure 7). Hydrogen participates in the reduction reaction of palladium oxide species (Equation (1)) [24,25]:

$$
\mathrm{H}_{2}+\mathrm{PdO} \rightarrow \mathrm{Pd}+\mathrm{H}_{2} \mathrm{O}
$$

It is often assumed, that irregular shape of reduction peak may results from overlapping palladium hydride decomposition at around $80^{\circ} \mathrm{C}$. In the case of the same sample (Pd/MCM-41) which was treated in argon at $400^{\circ} \mathrm{C}$ one can observe negative peak at $80^{\circ} \mathrm{C}$ due to hydrogen release from the decomposition of palladium hydride [25]. The absence of intensive peak at $60^{\circ} \mathrm{C}$ for samples treated in the argon flow confirms the formation of metallic palladium. The same curve was obtained

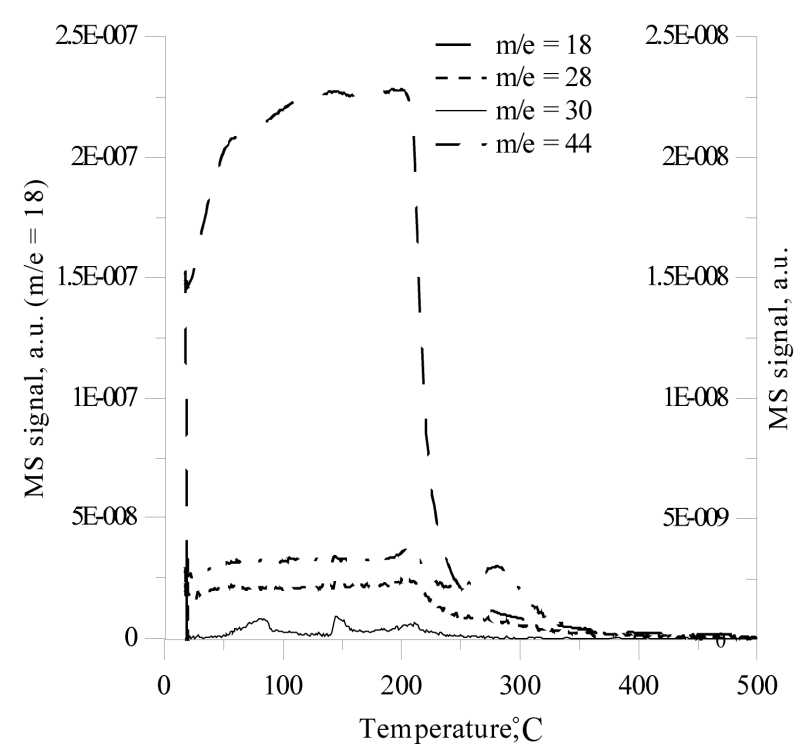

Figure 6. TPO curves of the Pd/MCM-41 sample. 


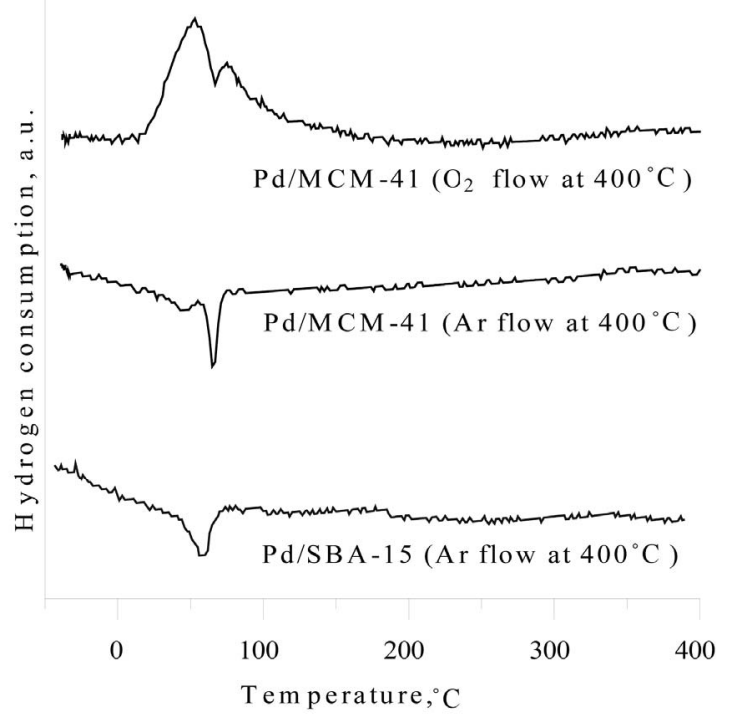

Figure 7. TPR curve of $\mathrm{Pd} / \mathrm{MCM}-41$ nanocomposite treated in oxygen at $400^{\circ} \mathrm{C}$ and argon flow at $400^{\circ} \mathrm{C}$ respectively.

for the sample Pd/SBA-15 calcined in argon flow at $400^{\circ} \mathrm{C}$.

Our previous investigations [21] suggested that presented method with the use of hydridesilica led to the formation of metallic palladium supported on silica matrix. This statement was assumed from the X-ray diffracttions studies of palladium-containing nanocomposites in the wide-angle region $\left(10^{\circ}-70^{\circ}\right)$. XRD pattern revealed three well-defined (111), (200) and (220) reflexes typical for the palladium face-centered cubic lattice [26]. However those reflexes were observed only for composite containing $1.5 \% \mathrm{Pd}$. For the samples with lower metal content $(0.5 \%)$ reflexes have very small intensity and almost invisible.

Palladium-containing nanocomposites synthesized using different supports, but the same metal content $(0.5 \%)$ were applied as catalysts in the oxidation of methane in the excess of oxygen.

The properties of catalysts are presented in the Table 2 and Figure 8. The best activity at low temperature can be observed for Pd/SBA-15 samples. While Pd/Silochrom samples shows higher activity at elevated temperatures.

It can be assumed from presented results, that, among the series of catalysts, the sample Pd/Silochrom seems to be the most active catalyst, upon which the complete conversion of methane was reached at the lowest temperature, i.e. $456^{\circ} \mathrm{C}$. It can be a result of accessibility of palladium nanoparticles for the reaction mixture. In the case of Pd/SBA-15 composite, there are spatial restrictions related with the fact that palladium nanoparticles are situated inside the pore channels of silica matrix. Should be noted that, despite the small content of metal, all the samples exhibit good catalytic activity.
Table 2. Characteristics of prepared palladium containing composites and their catalytic activity in methane oxidation reaction.

\begin{tabular}{ccc}
\hline Sample & $\begin{array}{r}\text { Diameter of Pd } \\
\text { nanoparticles, nm }\end{array}$ & $\begin{array}{c}\text { Temperature of } \\
\text { full methane } \\
\text { conversion, }{ }^{\circ} \mathrm{C}\end{array}$ \\
\hline Pd/Silochrom & $7-17$ & 456 \\
Pd/MCM-41 & $10-50$ & 477 \\
Pd/SBA-15 & 5 & 516 \\
\hline
\end{tabular}

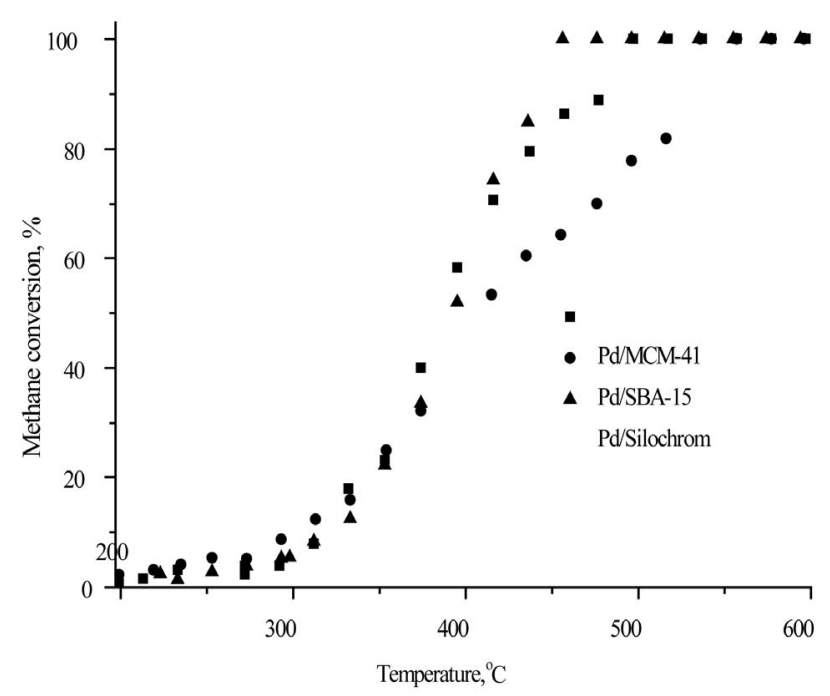

Figure 8. The activities of catalysts in the complete oxidation of methane.

\section{Conclusion}

Peculiarities of palladium nanoparticles synthesis on the surface of hydridesilica with different structural characteristics were studied. It was shown that modification of silica mesoporous support with silicon hydride groups may leads to the formation of small metallic palladium species. Their size can be related to the structural properties of the support. Thus, in the case with the usage of SBA-15 silica as support, formed nanoparticles are incorporated in the porous channels of matrix and have narrow pore size distribution. TPR studies suggest the formation of metallic palladium nanoparticles supported on silica. Synthesized palladium-containing nanocomposites were found to be effective catalysts in the reaction of methane oxidation. However, the activity of the catalysts, in spite of the presence of the small crystallites may be at a low level due to location and partial encapsulation of the active species in the pore channels.

\section{Acknowledgements}

This work was supported by European Community, seventh Framework Programm (FP/2007-2013), Marie 
Curie International Research Staff Exchange Scheme (grant no. 230790) and project MEC 06 MAT2006 01997.

\section{REFERENCES}

[1] L. J. Jongh, "Physics and Chemistry of Metal Clusters Compounds," Kluwer Academic Publishers, Dordrecht, 1994. doi:10.1007/978-94-015-1294-7

[2] I. Yuranov, P. Moeckli, E. Suvorova, P. Buffat, L. Kiwi-Minsker and A. Renken, " $\mathrm{Pd} / \mathrm{SiO}_{2}$ Catalysts: Synthesis of Pd Nanoparticles with the Controlled Size in Mesoporous Silicas," Journal of Molecular Catalysis A: Chemical, Vol. 192, No. 1-2, 2003, pp. 239-251. doi:10.1016/S1381-1169(02)00441-7

[3] L. X. Zhang, J. L. Shi, J. Yu, Z. L. Hua, X. G. Zhao and M. L. Ruan, "A New In-Situ Reduction Route for the Synthesis of Pt Nanoclusters in the Channels of Mesoporous Silica SBA-15," Advanced Materials, Vol. 14, No. 20, 2002, pp. 1510-1513. doi:10.1002/1521-4095(20021016)14:20<1510::AID-AD MA1510>3.0.CO;2-W

[4] C. Sener, T. Dogu and G. Dogu, "Effects of Synthesis Conditions on the Structure of Pd Incorporated MCM-41 Type Mesoporous Nanocomposite Catalytic Materials with High Pd/Si Ratio," Microporous and Mesoporous Materials, Vol. 94, No. 1-3, 2006, pp. 89-98. doi:10.1016/j.micromeso.2006.03.026

[5] D. D. Dharani and A. Sayari, "Applications of Pore-Expanded Mesoporous Silica 6. Novel Synthesis of Monodispersed Supported Palladium Nanoparticles and Their Catalytic Activity for Suzuki reaction," Journal of $\mathrm{Ca}$ talysis, Vol. 246, No. 1, 2007, pp. 60-65. doi:10.1016/j.jcat.2006.11.020

[6] P. Wang, Z. Wang, J. Li and Y. Bai, "Preparation, Characterizations, and Catalytic Characteristics of Pd Nanoparticles Encapsulated in Mesoporous Silica," Microporous and Mesoporous Materials, Vol. 116, No. 1-3, 2008, pp. 400-405. doi:10.1016/j.micromeso.2008.04.029

[7] J. Zhu, Z. Konya, V. F. Puntes, I. Kiricsi, C. X. Miao, J. W. Ager, A. P. Alivisatos and A. Somorjai, "Encapsulation of Metal (Au, Ag, Pt) Nanoparticles into the Mesoporous SBA-15 Structure," Langmuir, Vol. 19, No. 10, 2003, pp. 4396-4401. doi:10.1021/la0207421

[8] J. Garcia-Martinez, N. Linares, S. Sinibaldi, E. Coronado and A. Ribera, "Incorporation of Pd Nanoparticles in Mesostructured Silica," Microporous and Mesoporous Materials, Vol. 117, No. 1-2, 2008, pp. 170-177. doi:10.1016/j.micromeso.2008.06.038

[9] D. V. Goia and E. Matijevich, "Preparation of Monodispersed Metal Particles," New Journal of Chemistry, Vol. 22, No. 11, 1998, pp.1203-1215. doi:10.1039/a709236i

[10] J. J. Reed-Mundell, V. D. Nadkarni, M. J. Kunz, W. C. Fry and L. J. Fry, "Formation of New Materials with Thin Metal Layers through 'Directed' Reduction of Ions at Surface-Immobilized Silyl Hydride Functional Groups. Silver on Silica," Chemistry of Materials, Vol. 7, No. 9, 1995, pp. 1655-1660. doi:10.1021/cm00057a012

[11] G. B. Budkevich, V. J. Momot, I. I. Sirenko, J. A. Ta- rasenko and I. A. Sheka, "Reduction of Mercury Ions by Porous Highmolecular Hydridepolysiloxane," Ukrainian Chemistry Journal, Vol. 40, No. 10, 1974, pp. 364-368.

[12] G. B. Budkevich, I. B. Slinyakova and I. E. Neimark, "Reducing Properties of Xerogel of Hydridepolysiloxane," Colloid Journal, Vol. 28, No. 1, 1966, pp. 21-25.

[13] K. V. Katok, V. A. Tertykh and V. V. Yanishpolskii, "Synthesis and Application of Metal-Containing Silicas," In: A. Vaseashta and I. N. Mihailescu, Eds., NATO Science for Peace and Security Series B: Physics and Biophysics Functionalized Nanoscale Materials, Devices, and Systems, Springer, Berlin, 2008, pp. 335-339. doi:10.1007/978-1-4020-8903-9 27

[14] K. V. Katok, V. A. Tertykh and V. V. Yanishpolskii, "Reduction Nanoparticles of Gold in Surface Layer of Modified Silica," Russian Journal of Physical Chemistry A, Vol. 82, No. 9, 2008, pp. 1438-1441. doi:10.1134/S0036024408090045

[15] K. V. Katok, V. A. Tertykh and V. V. Yanishpolskii, "Synthesis and Application of Metal-Containing Silicas," In: J. P. Reithmaier, P. Petkov, W. Kulisch and C. Popov, Eds., NATO Science for Peace and Security Series B: Physics and Biophysics Nanostructured Materials for Advanced Technological Applications, Springer, Berlin, 2009, pp. 44-49.

[16] B. Stasinska, A. Machocki, K. Antoniak, M. Rotko, J. L. Figueiredo and F. Gonçalves, "Importance of Palladium Dispersion in $\mathrm{Pd} / \mathrm{Al}_{2} \mathrm{O}_{3}$ Catalysts for Complete Oxidation of Humid Low-Methane-Air Mixtures," Catalysis Today, Vol. 137, No. 2-4, 2008, pp. 329-334. doi:10.1016/j.cattod.2008.05.015

[17] B. Stasinska, W. Gac, T. Ioannides and A. Machocki, "Complete Oxidation of Methane over Palladium Supported on Alumina Modified with Calcium, Lanthanum, and Ce- rium Ions," Journal of Natural Gas Chemistry, Vol. 16, No. 4, 2007, pp. 342-348. doi:10.1016/S1003-9953(08)60002-X

[18] Y. H. Chin and D. E. Resasco, "Catalytic Oxidation of Methane on Supported Palladium under Lean Conditions: Kinetics, Structure and Properties," Royal Society of Chemistry-Catalysis, Vol. 14, 1999, pp. 1-39.

[19] M. Grun, K. K. Unger, A. Matsumoto and K. Tsutsumi, "Novel Pathways for the Preparation of Mesoporous Mcm-41 Materials - Control of Porosity and Morphology," Microporous and Mesoporous Materials, Vol. 27, No. 2-3, 1999, pp. 207-216. doi:10.1016/S1387-1811(98)00255-8

[20] D. Zhao, J. Feng, Q. Huo, N. Melosh, G. H. Frederickson, B. F. Chmelka and G. D. Stucky, "Triblock Copolymer Syntheses of Mesoporous Silica with Periodic 50 to 300 Angstrom Pores," Science, Vol. 279, No. 5350, 1998, pp. 548-552. doi:10.1126/science.279.5350.548

[21] N. A. Ivashchenko, K. V. Katok, V. A. Tertykh, V. V. Yanishpolskii and S. A. Khainakov, "Silica with Grafted Silicon Hydride Groups and Its Application for Preparation of Palladium Nanoparticles," International Journal of Nanoparticles, Vol. 4, No. 4, 2011, pp. 350358. doi:10.1504/IJNP.2011.043497

[22] V. N. Losev, G. V. Volkova, N. V. Maznyak, A. K. 
Trofimchuk and E. S. Yanovskaya, "Palladium Adsorption on Silica Modified with N-Allyl-N'-propylthiourea and Subsequent Determination by Spectrometry," Journal of Analytical Chemistry, Vol. 54, No. 12, 1999, pp. 1254-1258.

[23] N. A. Ivashchenko, K. V. Katok, V. A. Tertykh, V. V. Yanishpolskii, L. P. Oleksenko, L. V. Lutsenko and S. A. Khainakov, "Palladium Nanoparticles in the Surface Layer of Hydridesilica and Their Activity in Carbon Monoxide Oxidation," Kharkov University Bulletin, Chemical Series, Vol. 895, No. 41, 2010, pp. 241-247.

[24] C. W. Chou, S. J. Chu, H. J. Chiang, C. Y. Huang, C. J. Lee, S. R. Sheen, T. P. Perng and C. T. Yeh, "Tempera-
ture-Programmed Reduction Study on Calcination of Nano-Palladium," Journal of Physical Chemistry B, Vol. 105, No. 38, 2001, pp. 9113-9117. doi:10.1021/jp011170g

[25] K. Muto, N. Katada and M. Niwa, "Thermally-Stable Environmental Catalyst: Oxidation of Methane over Calcined Palladium Loaded on Silica Monolayer," Catalysis Today, Vol. 35, No. 1-2, 1997, pp. 145-151. doi:10.1016/S0920-5861(96)00141-1

[26] T. Teranishi and M. Miyake, "Size Control of Palladium Nanoparticles and Their Crystal Structures," Chemistry of Materials, Vol. 10, No. 2, 1998, pp. 594-600. doi:10.1021/cm9705808 